\title{
Caught in the Hotbox
}

\author{
Tina Sosa, MD*, Benjamin Kinnear, MD, MEd 1,2, Angela Y Choe, MD, MSc 3,4, Rabih Geha, MD5,6, David B Haslam, MD 2,7, \\ Pamela F Weiss, MD, MSCE ${ }^{8,9}$, Michelle W Parker, MD ${ }^{1,2}$
}

This icon represents the patient's case. Each paragraph that follows represents the discussants' thoughts.

${ }^{1}$ Division of Hospital Medicine, Cincinnati Children's Hospital Medical Center, Cincinnati, Ohio; ${ }^{2}$ Department of Pediatrics, University of Cincinnati College of Medicine, Cincinnati, Ohio; ${ }^{3}$ Division of Hospital Medicine, Children's Hospital of Los Angeles, Los Angeles, California; ${ }^{4}$ Department of Pediatrics, Keck School of Medicine, University of Southern California, Los Angeles, California; ${ }^{5}$ Department of Medicine, University of California, San Francisco, California; ' ${ }^{\circ}$ edical Service, San Francisco VA Medical Center, San Francisco, California; 'Division of Infectious Diseases, Cincinnati Children's Hospital Medical Center, Cincinnati, Ohio; ${ }^{8}$ Division of Rheumatology, Children's Hospital of Philadelphia, Philadelphia, Pennsylvania; ${ }^{9}$ Department of Pediatrics, Perelman School of Medicine, University of Pennsylvania, Philadelphia, Pennsylvania.

A 19-year-old woman presented to the emergency department (ED) with a 14-day history of progressive fevers, night sweats, abdominal pain, nonbloody and nonbilious vomiting, diarrhea, cough, and myalgia. The fever occurred daily with no noted temporal pattern, and she had no significant weight loss. The abdominal pain was diffuse and exacerbated by eating. She experienced multiple sporadic episodes of vomiting and diarrhea daily. She denied any rash or arthralgia.

She had no known medical history and took no medications. Family history was negative for autoinflammatory and autoimmune conditions. She had emigrated from Kenya to the United States 28 days ago. Her immunization status was unknown.

This patient has prolonged fevers and evidence of multisystem involvement. The most likely etiologic categories are infectious, inflammatory, rheumatologic, and neoplastic. For febrile patients who have recently emigrated to or travelled outside of the United States, it is important to consider common infections, as well as those endemic to the nation of exposure, which in this case includes malaria, typhoid fever, tuberculosis, cholera, acute viral hepatitis, chikungunya fever, dengue fever, yellow fever, and rickettsial disease. All of these, other than tuberculosis, commonly present with fever, vomiting, diarrhea, and myalgia. She may also have bacterial pneumonia or influenza given her fever and cough, although the chronicity and persistence of symptoms would be atypical. Acute infectious gastroenteritis is a common cause of fever, vomiting, and diarrhea. Most cases resolve in 7 to 10 days, so the duration raises suspicion for a nonviral etiology or an immunocompromised state.

Inflammatory causes could include the first presentation of inflammatory bowel disease (IBD), particularly if the patient

*Corresponding Author: Tina Sosa, MD; Email: Tina.sosa@cchmc.org; Twitter: @TinaKSosa.

Published online first April 20, 2021.

Received: January 17, 2020; Revised: May 19, 2020; Accepted: June 18, 2020

๑ 2021 Society of Hospital Medicine DOI 10.12788/jhm.3493 develops weight loss or eye, skin, or joint manifestations. The lack of rash or arthralgia makes rheumatologic conditions less likely. Prolonged fevers and night sweats could indicate malignancy such as intra-abdominal lymphoma, although infectious etiologies should be ruled out first.

Previously, on day 9 of symptoms, the patient presented to an ED at another institution. Laboratory evaluation at that time demonstrated an elevated aspartate aminotransferase (AST) level of $229 \mathrm{IU} / \mathrm{L}$ (reference, 0-40 IU/L) and alanine aminotransferase (ALT) level of $60 \mathrm{IU} / \mathrm{L}$ (reference, 0-32 IU/L) with normal alkaline phosphatase and bilirubin levels, proteinuria to $3+$ (normal, negative/trace), ketonuria to $2+$ (normal, negative), and hematuria to $2+$ (normal, negative). Complete blood count and electrolytes were normal. Computed tomography (CT) scans of the chest, abdomen, and pelvis with intravenous contrast were normal and without evidence of organomegaly.

AST and ALT elevations often indicate hepatocellular damage, although the normal bilirubin levels suggest normal hepatic function. Because CT may miss extrahepatic biliary pathology, a right upper quadrant ultrasound should be obtained to better evaluate patency of the biliary system and hepatic echotexture. Coagulation studies and viral hepatitis serology should be obtained. The disproportionate elevation of AST versus ALT can suggest alcohol use or nonhepatic etiologies such as myositis. Acute viral hepatitis is less likely given there is only mild to moderate elevation in aminotransferase levels. However, the remaining infectious etiologies can have this level of elevation and should still be considered.

Enteritis and IBD are still considerations despite the normal CT results. Transient asymptomatic hematuria or proteinuria can be seen in multiple conditions, particularly proteinuria with febrile illnesses. Urine microscopy to evaluate for casts could indicate a glomerular origin of the hematuria. First morning urine protein-to-creatinine ratio would help quantify the degree of proteinuria. Serum creatinine level should be measured to determine whether there is any renal dysfunction. 
While early imaging can be falsely negative, the unremarkable chest CT makes pneumonia and active pulmonary tuberculosis less likely.

Vital signs during this presentation were: temperature, $39.7^{\circ} \mathrm{C}$; heart rate, 126 beats per minute; blood pressure, 109/64 $\mathrm{mm} \mathrm{Hg}$; respiratory rate, 20 breaths per minute; and oxygen saturation, $98 \%$ on room air. She was ill-appearing, with diffuse abdominal tenderness without peritoneal signs. Other than her tachycardia, findings from her cardiopulmonary, neurologic, and skin exams were normal.

Laboratory testing revealed a white blood cell count of $4,300 / \mu \mathrm{L}$ (reference range, 4,500-13,000/ $\mu \mathrm{L}$ ), a hemoglobin level of $10.9 \mathrm{~g} / \mathrm{dL}$ (reference range, 11.7-15.7 $\mathrm{g} / \mathrm{dL}$ ) with a mean corpuscular volume of $77 \mathrm{fL}$ (reference range, 80-96 fL) and reticulocyte percentage of $0.5 \%$ (reference range, $0.5 \%$ $1.5 \%$ ), and a platelet count of $59,000 / \mu \mathrm{L}$ (reference range, $135,000-466,000 / \mu \mathrm{L})$. Her prothrombin time was $13.5 \mathrm{sec}-$ onds (reference range, 9.6-11.6 seconds) with an international normalized ratio of 1.3 (reference range, 0.8-1.1), erythrocyte sedimentation rate of $46 \mathrm{~mm} / \mathrm{h}$ (reference range, $0-20 \mathrm{~mm} / \mathrm{h}$ ), C-reactive protein level of $7.49 \mathrm{mg} / \mathrm{dL}$ (reference range, $<0.3$ $\mathrm{mg} / \mathrm{dL}$ ), and AST level of 194 units/L (reference range, 9-35 units/L). ALT, total and direct bilirubin, lipase, electrolytes, and creatinine levels were normal. An abdominal x-ray showed scattered air-fluid levels in a nonobstructed pattern.

Her mildly elevated prothrombin time and international normalized ratio suggest a coagulopathy involving either her extrinsic or common coagulation pathway, with disseminated intravascular coagulation (DIC) being most likely given her new thrombocytopenia and anemia. Hemolytic uremic syndrome and thrombotic thrombocytopenic purpura should be considered but would not cause coagulopathy. A peripheral smear to evaluate for schistocytes associated with microangiopathic hemolysis and serum fibrinogen to distinguish between DIC (low) and thrombocytopenic purpura or hemolytic uremic syndrome (normal or elevated) should be obtained. A thick and thin smear for malaria should also be performed.

Her new pancytopenia suggests bone marrow suppression or infiltration with or without a concomitant consumptive process such as sepsis with resulting DIC. Given her clinical picture, marrow infiltrative processes might include tuberculosis or malignancy, and marrow suppression may be caused by HIV or other viral infection. If she is found to have HIV, disseminated fungal or mycobacterial infections would become more likely. She now has an isolated elevated AST level, which could be secondary to hemolysis rather than hepatocyte damage. Findings from her abdominal exam are nonfocal; this is consistent with her x-ray findings, which reflect possible enteritis or colitis.

The most likely diagnosis currently is an infectious enteritis with resulting hematologic and hepatic abnormalities. Given her recent emigration from Kenya, typhoid fever and cholera are both possible, although cholera typically does not present with prolonged fever or severe abdominal pain. The severity and duration of her illness, and the abnormalities of her labora- tory findings, warrant empiric therapy with ceftriaxone to treat possible severe Salmonella enterica infection while awaiting blood and stool cultures.

The patient was admitted to the hospital and her symptoms continued. Results of serum HIV 1 and 2 polymerase chain reactions, herpes simplex virus 1 and 2 polymerase chain reactions, three malaria smears, human T-lymphotropic virus serologies, Toxoplasma serology, Bartonella serology, a stool culture, and multiple large volume blood cultures were negative. Serologic testing for hepatitis A, B, and C, Epstein-Barr virus, cytomegalovirus, and dengue virus were negative for acute infection. Results of an interferon-gamma release assay were indeterminate; results of purified protein derivative (PPD) and Candida antigen control testing were both negative. $\mathrm{Ce}-$ ruloplasmin and a1-antitrypsin levels were normal. An abdominal ultrasound showed central intrahepatic biliary duct dilatation, splenomegaly, and sluggish portal venous flow.

While central intrahepatic biliary ductal dilation could be caused by an obstructive lesion, none were seen on CT or ultrasound. Her normal alkaline phosphatase and bilirbuin levels also suggest functional patency of the biliary system. The thrombocytopenia, splenomegaly, and sluggish portal venous flow suggest possible portal hypertension, though no cirrhotic changes were noted on the ultrasound or abdominal CT. Her negative PPD and Candida antigen control results may suggest underlying immune dysregulation or suppression, though anergy could be secondary to sepsis.

Given her negative initial infectious evaluation, other etiologies such as atypical infections, rheumatologic disorders, and malignancies warrant consideration. She has no murmur; however, subacute bacterial endocarditis with a fastidious organism is possible, which could be investigated with a transthoracic echocardiogram. Other tests to consider include blood cultures for fungi and atypical mycobacterial species, and serology for Coxiella burnetii, chikungunya virus, and yellow fever. Rheumatologic conditions such as systemic lupus erythematosus, hemophagocytic lymphohistiocytosis $(\mathrm{HLH})$, or adult Still's disease should be considered. Complement levels and an antinuclear antibody panel, including those for dsDNA and Smith antigen, should be performed to evaluate for systemic lupus erythematosus. Serum ferritin, fibrinogen, and triglyceride levels should be measured to evaluate for HLH. Hematologic malignancy is also a consideration, particularly given her pancytopenia. Multicentric Castleman disease can cause prolonged fevers, pancytopenia, and elevated inflammatory markers, but is less likely without lymphadenopathy. A peripheral blood smear should be sent, and a bone marrow biopsy may be needed.

Empiric ciprofloxacin was initiated; however, the patient continued to have fevers up to $39.9^{\circ} \mathrm{C}$, abdominal pain, and myalgia. Ferritin level was over $3,000 \mathrm{ng} / \mathrm{mL}$ (reference range, $8-255 \mathrm{ng} / \mathrm{mL}$ ), and a soluble interleukin-2 (IL-2) receptor level was 1,188 units $/ \mathrm{mL}$ (reference range, $45-1,105$ units/ $\mathrm{mL})$. Triglycerides were normal. 
The elevated ferritin and soluble IL-2 levels raise concern for $\mathrm{HLH}$. Hyperferritinemia is relatively nonspecific because extremely elevated ferritin can be seen with other conditions, such as renal failure, hepatocellular injury, infection, rheumatologic conditions, and hematologic malignancy. Soluble IL-2 receptor elevation is more specific for HLH than ferritin or triglycerides, but alone does not make the diagnosis because it can be elevated in other rheumatologic disorders and malignancy. The HLH-2004 criteria are commonly used and require either molecular diagnostic testing or meeting at least five out of eight clinical and lab criteria to make the diagnosis. Our patient currently meets three criteria (fever, splenomegaly, and elevated ferritin). Elevated soluble IL-2 is part of the HLH-2004 criteria, but her level of elevation does not meet the required threshold $(\geq 2,400$ units $/ \mathrm{mL}$ ). Her cytopenias have also not quite met the HLH-2004 thresholds (two of the following three: hemoglobin $<9 \mathrm{~g} / \mathrm{dL}$, platelets $<100,000 / \mu \mathrm{L}$, and/or absolute neutrophil count $<1,000 / \mu \mathrm{L})$. Elevated aminotransferase levels and DIC are not part of the HLH-2004 criteria but are often seen with HLH.

Evaluation for an underlying infectious, rheumatologic, or malignant trigger should continue as previously discussed. If this patient does have HLH, it is most likely secondary to an infection, autoimmune disease, or malignancy rather than genetic HLH. HLH has a high mortality rate, but before beginning treatment with immunosuppressive agents, a peripheral smear and a bone marrow biopsy should be performed to evaluate for hematologic malignancy or signs of hemophagocytosis.

Empiric ciprofloxacin covers most bacterial etiologies of diarrhea, including those previously mentioned such as cholera and most strains of $S$ enterica. Her symptoms and laboratory findings (including cytopenias, elevated aminotransferases, and coagulopathy) could suggest enteric fever due to $S$ enterica serovar Typhi, which is endemic in Kenya. Results of blood and stool cultures, though negative, are relatively insensitive for this organism, particularly this far into the illness course. A bone marrow biopsy may also help with diagnosis of occult typhoid fever because marrow culture can be more sensitive than blood or stool culture.

\footnotetext{
A A bone marrow aspiration revealed hemophagocytic histiocytes, no malignant cells, and negative bacterial (including anaerobic), fungal, and acid-fast bacilli cultures. Considering the high mortality rate of untreated $\mathrm{HLH} /$ macrophage activation syndrome (MAS), empiric glucocorticoid administration was considered. However, this was withheld due to concern for ongoing undetected infection given her persistent fever and systemic symptoms.
}

There should still be high suspicion for HLH. Further evaluation for other laboratory manifestations of HLH such as fibrinogen and natural killer cell activity should be considered, as well as repeating her complete blood count to see if her cytopenias have progressed. Her marrow shows no evidence of hematologic malignancy, so other triggers of possible HLH should be sought out by continuing the workup. Consulting specialists from rheumatology and infectious disease may help clari- fy possible underlying diagnoses and the best management plan. If she continues to have organ damage or clinically worsens, it may be prudent to empirically broaden her antibiotic coverage and begin antifungal agents while starting glucocorticoid therapy for suspected $\mathrm{HLH}$.

A stool molecular screen from admission was returned positive for $\mathbf{S}$ enterica serovar Typhi. Ciprofloxacin was discontinued and ceftriaxone was started out of concern for antibiotic resistance. On hospital day 14, the patient's brother presented to the ED with fever. His blood and stool cultures were positive for $\boldsymbol{S}$ enterica serovar Typhi with intermediate sensitivity to ciprofloxacin and sensitivity to ceftriaxone. With continued treatment with ceftriaxone, the patient improved significantly. Following discharge, she remained afebrile and asymptomatic. During outpatient follow up, a repeat PPD was positive and she was diagnosed with and treated for latent tuberculosis.

\section{COMMENTARY}

The evaluation of a patient who has recently emigrated from a foreign nation requires a broad differential diagnosis and a keen awareness of the clinical conditions present in the patient's country of origin. This often involves knowledge of diseases infrequently encountered in daily practice, as well as awareness of the nuances of rare presentations and possible complications. When the presentation is not classic for a relevant infectious disease and clinical conditions from other diagnostic classes are considered, the evaluation and management of the patient is particularly challenging.

Typhoid fever is a severe systemic illness caused by the organism $S$ enterica serovar Typhi. The organism is ingested, penetrates the small intestinal epithelium, enters the lymphoid tissue, and disseminates via the lymphatic and hematogenous routes. Onset of symptoms typically occurs 5 to 21 days after ingestion of contaminated food or water. Clinical features include fever, chills, relative bradycardia (pulse-temperature dissociation), abdominal pain, rose spots (salmon-colored macules) on the trunk and abdomen, and hepatosplenomegaly. Diarrhea is not a typical symptom of patients with typhoid fever, which can lead to a delayed or missed diagnosis. Life-threatening complications can be seen, including gastrointestinal bleeding, intestinal perforation, and meningitis. ${ }^{1}$ Typhoid fever is most prevalent in impoverished areas with poor access to sanitation. Regions with the highest incidence include south-central Asia, southeast Asia, and southern Africa. ${ }^{2-4}$ Approximately 200 to 300 cases are reported in the United States each year. ${ }^{5}$

Classically, the diagnosis is made by means of clinical symptoms and a positive culture from a sterile site. A recent study of 529 patients found that $61 \%$ had positive blood cultures and $96 \%$ had positive bone marrow cultures. ${ }^{6}$ Our patient's diagnosis was significantly delayed by multiple negative cultures and failure to improve on first-line antibiotics, which initially suggested that the $S$ enterica serovar Typhi stool molecular screen likely represented carriage caused by colonization. Chronic $S$ enterica serovar Typhi carriage is defined as excretion of the 
organism in stool or urine 1 year or longer after acute infection. Rates of carriage range from $1 \%$ to $6 \%$, and up to $25 \%$ of carriers have no history of typhoid fever. ${ }^{1,7,8}$ Carriage is more common in females and in those with biliary tract abnormalities. ${ }^{9,10}$

Once a presumptive diagnosis is made, antibiotic choice remains a challenge. Resistance to fluoroquinolones, the preferred drug for multidrug-resistant typhoid fever, is growing but remains rare, at approximately 5\%.11,12 Ceftriaxone and azithromycin have been used successfully in areas with high resistance. ${ }^{13}$ Given the patient's slow response to therapy even after transitioning from ciprofloxacin to ceftriaxone, her brother's presentation and obtaining the antibiotic sensitivities for his organism were critical to confirming that our diagnosis and management decisions were correct.

One strongly considered diagnosis was HLH/MAS. MAS is an aggressive syndrome of excessive inflammation and tissue destruction caused by inappropriate immune system activation. It belongs to a group of histiocytic disorders collectively known as HLH. Aside from primary (genetic) forms, secondary forms exist that can be triggered by malignancy, infection, or rheumatologic disorders. In infection-associated HLH/MAS, viral infections are a common trigger, with Epstein-Barr virus being the most common. Association with bacterial infections, including tuberculosis and typhoid fever, has also been reported. ${ }^{14}$ Prompt therapy, often with immunosuppressive agents such as glucocorticoids, is essential for survival because there is a reported mortality rate of up to $50 \%$ when untreated. ${ }^{15}$ When infection-induced HLH/MAS occurs, treatment of the underlying infection is critical. ${ }^{14,15}$ The greatest barrier to a favorable outcome from HLH/MAS is often a delay in diagnosis because the rarity of this disease, the variable clinical presentation, and the lack of specificity of the clinical and laboratory findings make a conclusive diagnosis challenging.

In the presented case, diagnostic uncertainty challenged the decision to administer systemic glucocorticoids. Glucocorticoids conferred a risk of harm for multiple diagnoses that remained on the differential, including malignancy and infection. Her diagnostic evaluation made malignancy less likely, but because testing was unable to rule out tuberculosis as either the underlying cause or coinfection, the team opted to defer initiating glucocorticoids and instead closely monitor for a worsening inflammatory response. Following appropriate treatment of her systemic infection, her PPD was repeated and became positive. The negative PPD and Candida control obtained during her hospitalization were, therefore, likely caused by anergy in the setting of overwhelming systemic illness. Initiation of glucocorticoids prematurely in this case could have led to further harm because immunosuppression may have led to reactivation of latent tuberculosis or exacerbation of illness from an alternative but then undiagnosed infection.

The patient's ultimate unifying diagnosis was typhoid fever; however, there are mixed expert opinions as to whether the systemic immune activation was significant enough to merit the diagnosis of infection-induced secondary HLH/MAS. Despite the high morbidity and mortality that can accompany HLH/MAS, it has been reported that a significant proportion of cases of secondary HLH/MAS can be managed effectively with treatment of the underlying etiology; this may have been the case for our patient. ${ }^{14,15}$ The clinicians in this case were caught between diagnoses, unable to safely reach either one-much like a baseball player stranded between bases. Fortunately for this patient, the diagnosis ultimately emerged after a careful and thorough workup, assisted by the more straightforward diagnosis of her brother with the same disease.

\section{KEY TEACHING POINTS}

- Salmonella enterica serovar Typhi has a high false-negative rate in blood and stool cultures; therefore, clinical suspicion should remain high in the setting of a high pre-test probability.

- Fluoroquinolones are traditionally first-line therapy for typhoid fever, but the use of ceftriaxone and azithromycin is increasing because of rising fluoroquinolone resistance.

- Hemophagocytic lymphohistiocytosis/macrophage activation syndrome is characterized by excessive inflammation and tissue destruction caused by inappropriate immune system activation. This syndrome can be fatal without appropriate immunosuppressive therapy; however, glucocorticoid administration must be pursued with caution when infection and malignancy are on the differential diagnosis.

Disclosures: The authors have no conflicts of interest to disclose.

\section{References}

1. Parry CM, Hien TT, Dougan G, et al. Typhoid fever. $N$ Engl J Med. 2002;347(22):1770-1782. https://doi.org/10.1056/nejmra020201

2. Crump JA, Luby SP, Mintz ED. The global burden of typhoid fever. Bull World Health Organ. 2004;82(5):346-353.

3. Buckle GC, Walker CL, Black RE. Typhoid fever and paratyphoid fever: systematic review to estimate global morbidity and mortality for 2010. J Glob Health. 2012;2(1):010401. https://doi.org/10.7189/jogh.02.010401

4. Mogasale V, Maskery B, Ochiai RL, et al. Burden of typhoid fever in low-income and middle-income countries: a systematic, literature-based update with risk-factor adjustment. Lancet Glob Health. 2014;2(10):e570-e580. https://doi.org/10.1016/s2214-109x(14)70301-8

5. Lynch MF, Blanton EM, Bulens $S$, et al. Typhoid fever in the United States, 1999-2006. JAMA. 2009;302(8):859-865. https://doi.org/10.1001/ jama.2009.1229

6. Mogasale V, Ramani E, Mogasale W, Park J. What proportion of Salmonella typhi cases are detected by blood culture? a systematic literature review. Ann Clin Microbiol Antimicrob. 2016;15(1):32. https://doi.org/10.1186/s12941016-0147-z

7. Merselis JG Jr, Kaye D, Connolly CS, Hook EW. Quantitative bacteriology of the Typhoid carrier state. Am J Trop Med Hyg. 1964;13:425-429. https://doi. org/10.4269/ajtmh.1964.13.425

8. Lanata CF, Levine MM, Ristori C, et al. Vi serology in detection of chronic Salmonella typhi carriers in an endemic area. Lancet. 1983;2(8347):441-443. https://doi.org/10.1016/s0140-6736(83)90401-4

9. Lai CW, Chan RC, Cheng AF, Sung JY, Leung JW. Common bile duct stones: a cause of chronic salmonellosis. Am J Gastroenterol. 1992;87(9):1198-1199.

10. Hofmann E, Chianale J, Rollán A, Pereira J, Ferrecio C, Sotomayor V. Blood group antigen secretion and gallstone disease in the Salmonella typhi chronic carrier state. J Infect Dis. 1993;167(4):993-994. https://doi.org/10.1093/infdis/167.4.993

11. Steel AD, Hay Burgess DC, Diaz Z, Carey ME, Zaidi AKM. Challenges and opportunities for typhoid fever control: a call for coordinated action. Clin Infect Dis. 2016;62 (Suppl 1):S4-S8. https://doi.org/10.1093/cid/civ976

12. Hendriksen RS, Leekitcharoenphon P, Lukjancenko O, et al. Genomic signature of multidrug resistant Salmonella enterica serovar Typhi isolates related to a massive outbreak in Zambia between 2010 and 2012. J Clin Microbiol. 2015;53(1):262-272. https://doi.org/10.1128/jcm.02026-14

13. Crump JA, Sjölund-Karlsson M, Gordon MA, Parry CM. Epidemiology, clinical presentation, laboratory diagnosis, antimicrobial resistance, and antimicrobial management of Salmonella infections. Clin Microbiol Rev. 2015;28(4):901-936. https://doi.org/10.1128/cmr.00002-15

14. Rouphael NG, Talati NJ, Vaughan C, Cunningham K, Moreira R, Gould C. Infections associated with haemophagocytic syndrome. Lancet Infect Dis. 2007;7(12):814-822. https://doi.org/10.1016/s1473-3099(07)70290-6

15. Fisman DN. Hemophagocytic syndromes and infection. Emerg Infect Dis. 2000;6(6):601-608. https://doi.org/10.3201/eid0606.000608 